\title{
PERFIL DE FORMACIÓN DEL FUTURO TERAPEUTA OCUPACIONAL EN LA UNIVERSIDAD DEL VALLE
}

\section{* MISIÓN DE LA UNIVERSIDAD DEL VALLE}

La Universidad del Valle tiene como misión esencial educar mediante la generación y difusión de la ciencia, la cultura, el arte, la técnica, la tecnología, las humanidades y la filosofía, con una clara vocación de servicio a la sociedad. Así mismo, posibilita la formación integral del ser humano dentro de un espíritu creativo que permita el mejoramiento personal y el desarrollo de una sociedad democrática, tolerante y comprometida con los deberes civiles y los derechos humanos.

\section{* MISIÓN DEL PROGRAMA ACADÉMICO DE TERAPIA OCUPA- CIONAL}

Contribuir al desarrollo científico y tecnológico en el campo de la ocupación humana y sus disfunciones, con'una responsable actitud de servicio a la población con deficiencia, discapacidad o minusvalía o en riesgo de adquirirla y a la sociedad en general, a través de la formación integral de los educandos como personas autónomas y creativas, para que con espíritu analítico y crítico escojan las alternativas teóricas y prácticas encaminadas a su crecimiento personal y al desarrollo de una sociedad más justa, equitativa y democrática.

\section{* historia del pROGRAMA ACADÉmICO:}

El Plan de estudios de Terapia Ocupacional en la Universidad del Valle, se inició en el segundo semestre del año de 1986, por la gestión e impulso del Dr. Jaime Villaquirán y la tesis realizada durante la maestría en administración educativa de la Terapeuta Ocupacional Nohra de Bastidas, la cual sirvió como base para el curriculum inicial del Plan de Estudios.

Este Plan, así como el de fisioterapia y fonoaudiología, en su comienzo hacían parte del Departamento de Medicina Física y Rehabilitación, ubicado locativamente en el Hospital Universitario del Valle. 
Posteriormente, la Universidad y más específicamente la Facultad de Salud, inicia una reforma académica y administrativa, dando origen a las Escuelas. Se crea la Escuela de Rehabilitación Humana, con sus Departamentos de Fisiocinética Humana, Ocupación Humana y Fonoaudiología y con los tres Programas Académicos de Fisioterapia, Terapia Ocupacional y Fonoaudiología.

Actualmente el desarrollo del Programa Académico ha sido objeto de la reforma académica y se ha repensado al nuevo profesional que demanda la sociedad actual, ofreciendo un nuevo proyecto educativo enmarcado en los principios de dicha reforma curricular.

En la Escuela de Rehabilitación Humana, se ha creado el Grupo de cátedra de rehabilitación, el cual ha incorporado la docencia, investigación y la proyección social a todos los Programas y Departamentos. Inicialmente se trabaja con asignaturas interdisciplinarias, tales como Discapacidad y sociedad, discapacidad y rehabilitación, integración escolar y laboral, entre otras.

\section{* OBJETIVOS}

El Programa Académico está compuesto por dos ciclos: uno de fundamentación que corresponde a los cuatro primeros semestres y el pro. fesional que corresponde a los últimos seis semestres.

\section{CICLO DE FUNDAMENTACIÓN}

\section{OBJETIVOS GENERALES}

Al finalizar el ciclo de fundamentación el estudiante estará en capacidad de:

1. Analizar integralmente el ser humano que utiliza la ocupacion como medio de expresión y autorrealización en sus dimensiones psicosocial, biológica, cultural, humanística y económica.

2. Asumir una actitud de compromiso ético flexible frente a la heterogeneidad y diversidad de factores que determinan la ocupación humana. 


\section{OBJETIVOS ESPECÍFICOS:}

1. Reconocer los elementos filosóficos, éticos $y / 0$ artísticos del hombre como sistema abierto.

2. Reconocer los aspectos biosicosociales que influyen en la ocupación del ser humano durante su ciclo vital.

3. Ubicar al individuo dentro de su contexto socioeconómico, cultural y humanístico para que se desarrolle a través de la ocupación.

4. Actuar con razonamiento y argumento frente a la realidad del ser humano y su desempeño ocupacional a lo largo de su vida.

5. Reconocer los avances e implicaciones del desarrollo tecnológico en el quehacer profesional del Terapeuta Ocupacional.

6. Acercarse a la comprensión de la persona con discapacidad y su relación con el entorno.

\section{CICLO PROFESIONAL}

\section{OBJETIVO GENERAL}

El estudiante de Terapia Ocupacional estará en capacidad de utilizar la metodología científica en la solución de problemas relacionados con el desempeño ocupacional del individuo, ya sea funcional, disfuncional o en riesgo de adquirir la disfunción.

\section{OBJETIVOS ESPECÍFICOS}

Al finalizar el ciclo profesional el estudiante estará en capacidad de:

1. Conocer la reglamentación gubernamental, las políticas y como afectan la provisión de servicios de Terapia Ocupacional.

2. Integrar la investigación, la teoría y la práctica para el desarrollo de la praxis profesional con calidad. 
3. Describir la compleja naturaleza del ser humano biosicosocial y sus necesidades ocupacionales en una perspectiva de sistemas.

4. Explicar la naturaleza transaccional de la persona, medio ambiente cultura y la influencia de cada uno en el desempeño ocupacional.

5. Integrar los conceptos de ciencias sociales, naturales, filosóficas, tecnológicas, humanísticas, matemáticas' y artísticas al desempeño ocupacional

6. Identificar en el desempeño ocupacional los componentes interpersonales y medioambientales para ser utilizados en prevención y mediación de la disfunción y/o facilitación de la adaptación para lograr su desempeño ocupacional.

7. Interferir los factores del entorno biológico y sicosocial que puedan interrumpir el desempeño ocupacional de las personas en cualquier etapa de su vida.

8. Utilizar la ocupación como medio de promoción para el desarrollo de competencias del individuo dentro de su entorno.

9. Analizar el uso de varias perspectivas teóricas de Terapia Ocupacional y como afectan el desempeño ocupacional en el trabajo, el juego y el autocuidado y el tiempo libre.

10. Desarrollar programas de intervención basados en la racionalización holística de la interacción de la persona en su entorno.

11. Establecer una intervención holística, basada en metas y planes sobre los intereses únicos individuales y las necesidades específicas de la persona, la cultura y las políticas gubernamentales, basados en los marcos de referencia de Terapia Ocupacional.

12. Participar en la promoción de Terapia Ocupacional a través de organizaciones profesionales, gubernamentales y de proyección social. 


\section{* ÉNFASIS DEL PROGRAMA ACADÉMICO DE TERAPIA OCUPACIONAL EN LA UNIVERSIDAD DEL VALLE}

\section{OPCIÓN SOCIAL}

La Universidad del Valle con el planteamiento de la Reforma Curricular en su acuerdo 001 de 1993 pretende lograr cambios cualitativos en la formación integral de los estudiantes.

El Programa Académico de Terapia Ocupacional enfatiza su posición en la opción social, porque maneja la ocupación en sus componentes biológico, psicosocial, cognitivo y medioambiental y su función primordial es promover el bienestar ocupacional. Prevenir, intervenir y rehabilitar en casos de deficiencia, discapacidad o minusvalía, con el propósito de lograr la autorrealización personal en su desempeño ocupacional.

La Terapia Ocupacional, cuyo objetivo de estudio es la «ocupación humana» en sus dimensiones de autocuidado, juego y trabajo, va dirigida a personas, con riesgo de adquirir una disfunción, que le impida o dificulte operar funcionalmente dentro de su medio ocupacional.

La profesión tiene tres finalidades:

* Comprender la naturaleza ocupacional

* Explicar el valor intrínseco de la ocupación

* Sustentar el potencial de las ocupaciones.

El Programa Académico de Terapia Ocupacional, en la Universidad del Valle, está sustentado en el marco de la opción social, puesto que concibe al hombre a través de su vida en una interacción dinámica entre su desempeño ocupacional como una fuerza de desarrollo humano y su relación con él. 


\title{
* eStructura CURRICULAR DEL PROGRAMA ACADÉmico DE TERAPIA OCUPACIONAL
}

\author{
ESTRUCTURA BASICA
}

\begin{tabular}{|c|c|}
\hline CICLO DE FUNDAMENTACION & CICLO PROFESIONAL \\
2 AÑOS & 3 AÑOS \\
\hline Obligatorias de la opción & Asignaturas de la profesión \\
OBO & AP \\
\hline Obligatorias de la profesión & Asignaturas electivas \\
OBP & AE \\
\hline Electivas complementarias & \\
EC & \\
\hline
\end{tabular}

\section{CICLOS DE FORMACIÓN}

\section{* CICLO DE FUNDAMENTACIÓN}

Proporciona el dominio de los conceptos, métodos y operaciones en uno - diversos campos del saber; para garantizar la formación académica bási$\mathrm{ca}$, propia de quienes aspiran a ejercer con solvencia científica y humana una profesión universitaria. Este ciclo favorece el espíritu argumentativo, la capacidad para razonar y relacionar conceptos, contextos y problemas disímiles en apariencia y el desarrollo del espíritu crítico y la capacidad de valoración.

Ofrece al estudiante un espectro de alternativas de educación que le faciliten su opción profesional y le procuren una formación integral:

Tiene una duración de dos años e ingresan a él los estudiantes que cumplan con el puntaje ICFES, mínimo 310 . El estudiante cursa en promedio 18 créditos por semestre, distribuidos en materias de cada componente.

Durante este ciclo los estudiantes deben cursar asignaturas que tienen lós componentes de Artes, Humanidades, Filosofía, Matemáticas, Tecnolo- 
gía, Ciencias Naturales y Sociales, los cuales tienen su diferencia básica en la distribución de créditos para cada uno. Por lo tanto la distribución se hace en componentes de la opción (OBO), de la profesión (OBP) y en electivas complementarias (EC). Además cursan materias obligatorias de Ley (OL): Deporte, Constitución Política de Colombia, Español y Examen de Proficiencia en idioma extranjero, de acuerdo a la siguiente tabla:

\begin{tabular}{|c|c|c|c|}
\hline 01 & 02 & 03 & 04 \\
\hline \multicolumn{4}{|c|}{ ASIGNATURAS OBLIGATORIAS DE LA OPCIÓN SOCIAL } \\
\hline \multicolumn{4}{|c|}{$\begin{array}{llll}\text { - matemáticas } & 3 \text { créd. } & \text { - filosófica } & 8 \text { créd. } \\
\text { - científica natural } & 9 \text { créd. } & \text { - social } & 12 \text { créd. } \\
\text { - tecnológico } & 7 \text { créd. } & & \end{array}$} \\
\hline \multicolumn{4}{|c|}{ ASIGNATURAS ELECTIVAS } \\
\hline \multicolumn{4}{|c|}{$\begin{array}{l}\text { - artísticas } \\
\text { - humanísticas } 12 \text { créd. }\end{array}$} \\
\hline \multicolumn{4}{|c|}{ ASIGNATURAS OBLIGATORIAS DE LA PROFESIÓN 21 Créd. } \\
\hline Discapacidad y sociedad & Discapacidad y rehabilitación & Conceptualizac. ocup. hum & n. Productividad en el desempeño \\
\hline Fundamentos del juego & & Fundam. económ. ocupac. & Desarrollo Humano \\
\hline
\end{tabular}

\section{* CICLO PROFESIONAL:}

Está orientado a la formación sistemática en el campo profesional específico. Conjuntamente con el Ciclo de Fundamentación, contribuye a la formación académica del egresado.

El estudiante cursa asignaturas de la profesión (AP) y asignaturas electivas $(\mathrm{AE})$, las cuales están orientadas a complementar aspectos diferentes a su profesión a profundizar en su campo disciplina. Las selecciona a partir de la oferta de la Universidad y de su área de interés. Durante el Ciclo Profesional, debe cursar 18 créditos de AE. 


\section{CICLO PROFESIONAL}

\begin{tabular}{|l|l|l|l|l|l|}
\hline \multicolumn{1}{|c|}{05} & \multicolumn{1}{c|}{06} & \multicolumn{1}{c|}{07} & \multicolumn{1}{c|}{08} & \multicolumn{1}{c|}{09} & \multicolumn{1}{c|}{10} \\
\hline $\begin{array}{l}\text { Conceptualizacion de } \\
\text { TO }\end{array}$ & Modelos de TO & $\begin{array}{l}\text { Procesos evaluativos } \\
\text { en TO }\end{array}$ & $\begin{array}{l}\text { Práctica de } \\
\text { Prevención }\end{array}$ & $\begin{array}{l}\text { Práctica } \\
\text { en dos sectores }\end{array}$ & $\begin{array}{l}\text { Práctica en el tercer } \\
\text { sector }\end{array}$ \\
\hline $\begin{array}{l}\text { Análisis del } \\
\text { desempeño }\end{array}$ & $\begin{array}{l}\text { Desarrollo } \\
\text { Empresarial }\end{array}$ & TO en Salud I & TO en Salud II & & $\begin{array}{l}\text { Práctica de } \\
\text { profundización }\end{array}$ \\
\hline Neuroanatomía & Patología & TO en Educación I & TO en Educación II & & 1 \\
\hline Fisiologja & Neurofisiologia & TO en Laboral I & TO en Laboral II & & \\
\hline Psicopatología & & Investigación I & Investigación II & Investigación III & Trabajo de Grado \\
\hline
\end{tabular}

DURACIÓN DE LA CARRERA: 10 SEMESTRES (5 AÑOS)

\section{* NÚMERO DE EGRESADOS}

Desde el año de 1990, en el cual salió la primera promoción, hasta el presente año, hay 150 egresados Terapeutas Ocupacionales.

\section{* PRÁCTICAS}

Los estudiantes deben realizar tres niveles de práctica, así:

1. Práctica preventiva. Los estudiantes realizan una práctica durante todo el semestre. El énfasis es la prevención de los factores intrínsecos o extrínsecos que puedan alterar el desempeño ocupacional de las personas en sus diferentes contextos. La cursan en octavo semestre y es prerrequisito para el siguiente nivel de práctica.

2. Práctica de intervención. Realizan práctica en el sector educativo (integración escolar y educación regular), en el sector laboral (salud ocupacional y programas de higiene y seguridad industrial) y en el sector salud (salud mental, física y ancianos). Cada práctica tiende una duración de nueve semanas, con una intensidad de 20 horas presenciales, más trabajo independiente, el cual realiza el estudiante. Las cursan en noveno y la mitad de décimo semestre. Es prerrequisito para el tercer nivel de práctica.

3. Práctica de profundización. El estudiante realiza una práctica seleccionada por él, a partir de su interés y con base en el proyecto de grado. Una modalidad de ejecución es la pasantía, la cual apoya la creación de nuevas fuentes de trabajo, desde la academia. Tienen una duración de 
nueve semanas, con una intensidad de 40 horas presenciales.

\section{SITIOS DE PRÁCTICA}

Actualmente los estudiantes de noveno semestre, realizan su práctica por los siguientes lugares:

* SALUD: Hospital Universitario del Valle, Unidad de Salud Mental y Departamento de Medicina Física y rehabilitación y Ancianato Cottolengo

* LABORAL: Universidad del Valle y Smurfit Cartón de Colombia.

* EDUCATIVA: Colegio Eustaquio Palacios y CENDOE.

\section{Claudia María Payán Villamizar}

Directora, Programa Académico

Terapia Ocupacional

Santiago de Cali, septiembre de 1997 